\title{
Global Optimization by Basin-Hopping and the Lowest Energy Structures of Lennard-Jones Clusters Containing up to 110 Atoms
}

\author{
David J. Wales \\ University Chemical Laboratories, Lensfield Road, Cambridge CB2 1EW, UK \\ Jonathan P. K. Doye \\ FOM Institute for Atomic and Molecular Physics, Kruislaan 40\%, \\ 1098 SJ Amsterdam, The Netherlands
}

\begin{abstract}
We describe a global optimization technique using 'basin-hopping' in which the potential energy surface is transformed into a collection of interpenetrating staircases. This method has been designed to exploit the features which recent work suggests must be present in an energy landscape for efficient relaxation to the global minimum. The transformation associates any point in configuration space with the local minimum obtained by a geometry optimization started from that point, effectively removing transition state regions from the problem. However, unlike other methods based upon hypersurface deformation, this transformation does not change the global minimum. The lowest known structures are located for all Lennard-Jones clusters up to 110 atoms, including a number that have never been found before in unbiased searches.
\end{abstract}

\section{INTRODUCTION}

Global optimization is a subject of intense current interest 1 Improved global optimization methods could be of great economic importance, since improved solutions to travelling salesman type problems, the routing of circuitry in a chip, the active structure of a biomolecule, etc., equate to reduced costs or improved performance. In chemical physics the interest in efficient global optimization methods stems from the common problem of finding the lowest energy configuration of a (macro)molecular system. For example, it seems likely that the native structure of a protein is structurally related to the global minimum of its potential energy surface (PES). If this global minimum could be found reliably from the primary amino acid sequence, this knowledge would provide new insight into the nature of protein folding and save biochemists many hours in the laboratory. Unfortunately, this goal is far from being realized. Instead the development of global optimization methods has usually concentrated on much simpler systems.

Lennard-Jones (LJ) clusters represent one such test system. Here the potential is

$$
E=4 \epsilon \sum_{i<j}\left[\left(\frac{\sigma}{r_{i j}}\right)^{12}-\left(\frac{\sigma}{r_{i j}}\right)^{6}\right],
$$

where $\epsilon$ and $2^{1 / 6} \sigma$ are the pair equilibrium well depth and separation, respectively. We will employ reduced units, i.e. $\epsilon=\sigma=1$ throughout. Much of the initial interest in LJ clusters was motivated by a desire to calculate nucleation rates for noble gases. However, as a result of the wealth of data generated, the LJ potential has been used not only for studying global optimization but also the effects of finite size on phase transitions such as melting. Through the combined efforts of many workers likely candidates for the global mining of $\mathrm{LJ}_{N}$ clusters have been found up to $N=147.216$ This represents a significant achievement since extrapolation of Tsai and Jordan's comprehensive enumeration of minima for small LJ clusters 17 suggests that the PES of the 147-atom cluster possesses of the order of $10^{60}$ minima 18

Previous studies have revealed that the Mackay icosahedron 19 provides the dominant structural motif for LJ clusters in the size range 10-150 atoms. Complete icosahedra are possible at $N=13,55,147, \ldots$ At most intermediate sizes the global minimum consists of a Mackay icosahedron at the core covered by a low energy overlayer. As a consequence of the phase behaviour of LJ clusters, finding these global minima is relatively easy. Studies have shown that in the region of the solid-liquid transition the cluster is observed to change back and forth hetween a liquid-like form and icosahedral structures 20,21 As a result of this 'dynamic coexistence' a method as crude as molecular dynamics within the melting region coupled with systematic minimization of configurations generated by the trajectory is often sufficient to locate the global minimum 22
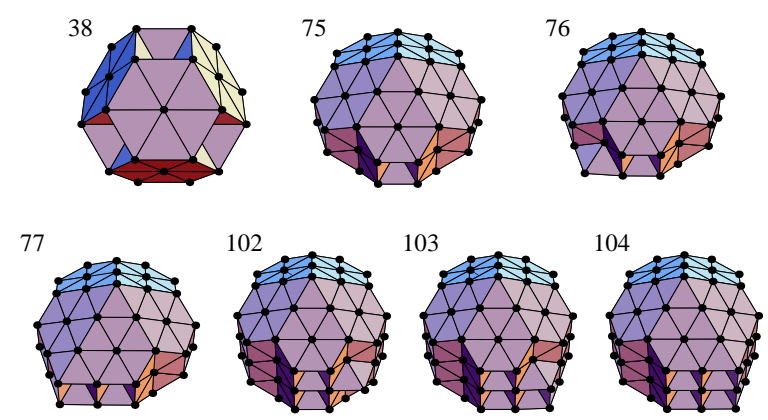

FIG. 1. Non-icosahedral Lennard-Jones global minima. 
However, there are a number of sizes at which the global minimum is not based on an icosahedral structure. These clusters are illustrated in Figure 1. For LJ $\mathrm{J}_{38}$ the lowest energy structure is a face-centred-cubic (fcc) truncated octahedron, 13,14 and for $N=75,76,77,102$, 103 and 104 geometries based on Marks' decahedra 23 are lowest in energy.14,15 For these cases finding the lowest minimum is much harder because the global minimum of free energy only becomes associated with the global potential energy minimum at temperatures well below melting where the dynamics of structural relaxation are very slow. For $\mathrm{LJ}_{38}$, the microcanonical temperature for the transition from face-centred cubic to icosahedral structures has been estimated to be about $0.12 \epsilon k^{-1}$, where $k$ is the Boltzmann constant, and for $\mathrm{LJ}_{75}$ the estimate for the decahedral to icosahedral transition is about $0.09 \epsilon k^{-1} .24$ (For comparison, melting typically occurs at about $T=0.2-0.3 \epsilon k^{-1}$.)

The topography of the PES can also play a key role in determining the ease of global optimization 25. A detailed study of the $\mathrm{LJ}_{38}$ PES has shown that there is a large energ barrier between the fcc and icosahedral structure 26 which correspond to well-separated regions of the PES. Furthermore, fcc and decahedral structures have less polytetrahedral character than icosahedral structures, and hence they have less in common with the liquid-like state, which ischaracterized by disordered polytetrahedral packing.27 29 Since the vast majority of configuration space is dominated by 'liquid-like' configurations, it is therefore harder to find global minima based upon fcc and decahedral packing using unbiased searches.

These considerations explain why global optimization methods have only recently begun to find the truncated octahedron 13.62031 and why, until now, the Marks' decahedron has never been found by an unbiased global optimization method. The greater difficulty of finding the $\mathrm{LJ}_{75}$ global minimum compared to $\mathrm{LJ}_{38}$ can probably be explained by the slightly smaller transition temperature, the sharper transition and the much larger number of minima on the $\mathrm{LJ}_{75}$ PES.

Before we consider the effectiveness of different global optimization methods for Lennard-Jones clusters it is interesting to note that the use of physical principles to construct good candidate structures for the global minima 25 814,15 or to redpes the configuration space that needs to be searched 1012 led to the initial discovery of $93 \%$ of the LJ global minima in this size range. It seems that physical insight into a specific problem will often be able to beat unbiased global optimization, a view expressed by Ngo et al.32 in their discussion of computational complexity.

One difficulty in evaluating the relative performances of different global optimization methods is that, too often, the methods have only been applied to small clusters, or to larger clusters with global minima that are especially stable, such as $\mathrm{LJ}_{55}$. It is also difficult to draw any firm conclusions about how efficient different methods may be when the number of searches employed varies widely. However, it seems reasonable to suggest two hurdles that any putative global optimization approach should aspire to. The first is the location of the truncated octahedron for $\mathrm{LJ}_{38}$, and any method which fails this test is unlikely to be useful. The second hurdle is the location of the Marks decahedron for $\mathrm{LJ}_{75}$; this problem poses a much more severe test for an unbiased search and one which does not appear to have been passed until the present work.

The most successful global optimization results for LJ clusters reported to date are for genetic algorithms.16. 30.33,34 These methods mimic some aspects of biological evolution: a population of clusters evolves to low energy by mutation and mating of structures, and selection of those with low potential energy. To be successful new configurations produced by 'genetic manipulation' are mapped onto minima by a local optimization algorithm such as the conjugate gradient method. The study by Deaven et al. is particularly impressive, since these workers matched or beat all the lowest energy minima that they knew of up to $N=100$, including the truncated octahedron (although they probably missed the global minima at $N=69$ and 75-78). Niesse and Mayne were also able to locate the $\mathrm{LJ}_{38}$ truncated octahedron, and report that this structure took about 25 times longer to find than the icosahedral global minima of the neighbouring sizes.

Another class of global optimization techniques, sometimes called hypersurface deformation methods, attempts to simplify the problem by applying a transformation to the PES which smoothes it and reduces the number of local minima 35,36 The global minimum of the deformed PES is then mapped back to the original surface in the hope that this will lead back to the global minimum of the original PES. The distinctions between the various methods of this type lie in the type of transformations that are used, which include applying the diffysion equation, 37.38 increasing the range of the potential, 39,40 and shifting the position of the potential minimum towards the origin 41 The performance of hypersurface deformation methods has been variable: the potential shift approach managed to find the 38-atom truncated octahedron, but other workers report difficulties 37,38 for the trivial cases of $\mathrm{LJ}_{8}$ and $\mathrm{LJ}_{9}$ where there are only 8 and 21 minima on the PES, respectively.

Although intuitively appealing, the problem with hypersurface deformation is that there is no guarantee that the global minimum on the deformed PES will map onto the global minimum of the original surface. This difficulty is clearly illustrated when we consider Stillinger and Stillinger's suggestion of increasing the range of the potential:39 it has been shown that the global minimum may in fact depend rather sensitively on the range of the potential, with the appearance of numerous 'rangeinduced' transitions. 14.42

Other methods include those based on 'annealing'. Such approaches take advantage of the simplification in the free energy landscape that occurs at high tempera- 
tures, and attempt to follow the free energy global minimum as the temperature is decreased. At zero Kelvin the free energy global minimum and the global minimum of the PES must coincide. Standard simulated annealing 43 was used by Wille to find a few new minima at small sizes 1 but does not appear to have been systematically applied to LJ clusters. More sophisticated variants of this technique include gaussian density annealing and analggyes, 44 but again some appear to fail at small sizes.46,47

The difficulty with the annealing approach methods is that, if the free energy global minimum changes at low temperatures where dynamical relaxation is slow, the algorithms will become stuck in the structure corresponding to the high temperature free energy global minimum. Such methods are therefore likely to experience difficulties in finding the global minima for $\mathrm{LJ}_{38}$ and $\mathrm{LJ}_{75}$. In the language employed in recent protein folding literature 48 annealing will fail when $T_{f}<T_{g}$, where $T_{f}$ is the 'folding' temperature below which the global potential energy and free energy minima coincide, and $T_{g}$ is the 'glass' temperature at which the system effectively becomes trapped in a local minimum.

Another method which attempts to reduce the effects of barriers on the PES makes use of quantum tunnelling. The diffusion Monte Carlo approach is used to find the ground state wavefunction, which should become localized at the global minimum as $\hbar$ is decreased to zero. 49 A more rigorous approach has been applied by Maranas and Floudas, who found upper and lower bounds for the energy of the global minimum. However, the computational expense of this method, which scales as $2^{N}$ with the number of atom-means that it has only been used for small systems 50,51 Most of the above stydies, along with the recently described 'pivot method'"52 54 and 'taboo search' 55, 56 have yet to prove their usefulness by passing the first hurdle for $\mathrm{LJ}_{38}$ suggested above. However, this does not necessarily mean that these approaches should be discounted, since some authors have only applied their algorithms to smaller clusters and may not have run enough searches to achieve convergence.

In the present work we present the results of a 'basinhopping' global optimization technique for LennardJones clusters. All the known lowest energy structures up to $N=110$ have been located successfully, including three minima not previously reported. (See Tables 1 and 2.) This method is also the first unbiased algorithm to find the global minima based on the Marks decahedron around $\mathrm{LJ}_{75}$ and $\mathrm{LJ}_{102}$. For reference, we collect the rather scattered results previously reported for LJ clusters to provide a complete catalogue of the energies and point groups of the lowest energy minima that we know of. The results have been collected in the first entry of the Cambridge Cluster Database at http://brian.ch.cam.ac.uk.

\section{METHOD}

The present approach has been guided by previous work on energy landscapes which has identified features that enable the system to locate its global minimum efficiently 48 In particular, analysis of model energy landscapes using a master equation approach for the dynamics, has provided good evidence that such a surface should have a large potential energy gradient and the lowest pessible transition state energies or rearrangement barriers 25 These results immediately suggest a simple way to transform the PES which does not change the global minimum, nor the relative energies of any local minima. We consider the transformed energy $\tilde{E}$ defined by:

$$
\tilde{E}(\mathbf{X})=\min \{E(\mathbf{X})\}
$$

where $\mathbf{X}$ represents the $3 N$-dimensional vector of nuclear coordinates and min signifies that an energy minimization is performed starting from $\mathbf{X}$. In the present work energy minimizations were performed using the PolakRibiere variant of the conjugate gradient algorithm.57 Hence the energy at any point in configuration space is assigned to that of the local minimum obtained by the given geometry optimization technique, and the PES is mapped onto a set of interpenetrating staircases with plateaus corresponding to the set of configurations which lead to a given minimum after optimization. A schematic view of the staircase topography that results from this transformation is given in Figure 2. These plateaus, or basins of attraction, have been visualized in previous work as a means to compare the efficiency of different transition state searching techniques.5859

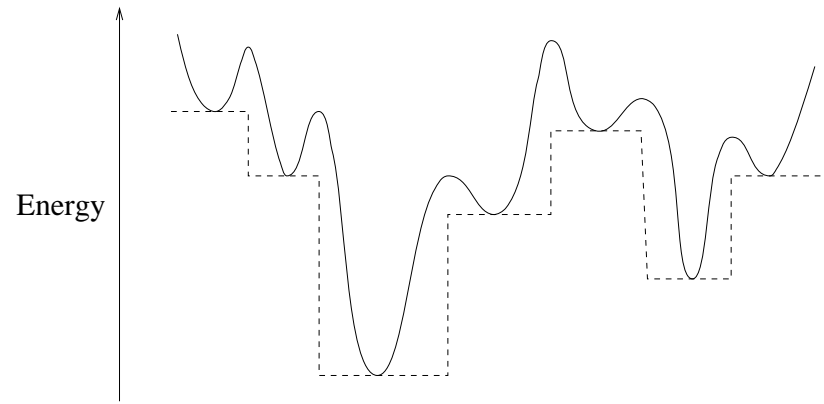

FIG. 2. A schematic diagram illustrating the effects of our energy transformation for a one-dimensional example. The solid line is the energy of the original surface and the dashed line is the transformed energy, $\tilde{E}$.

The energy landscape for the function $\tilde{E}(\mathbf{X})$ was explored using a canonical Monte Carlo simulation at a constant reduced temperature of 0.8 . At each step all coordinates were displaced by a random number in the range $[-1,1]$ times the step size, which was adjusted to give an acceptance ratio of 0.5 . The nature of the transformed surface allowed relatively large step sizes of between $0.36-0.40$. For each cluster in the range considered 
seven separate runs were conducted. Five of these each consisted of 5000 Monte Carlo steps starting from different randomly generated configurations of atoms confined to a sphere of radius 5.5 reduced units. The subsequent geometry optimizations employed a container of radius one plus the value required to contain the same volume per atom as the fcc primitive cell. The container should have little effect on any of our results and is only required to prevent dissociation during the conjugate gradient optimizations.

The convergence criterion employed for the conjugate gradient optimizations used in the Monte Carlo moves need not be very tight. In the present work we required the root-mean-square (RMS) gradient to fall below 0.01 in reduced units and the energy to change by less than $0.1 \epsilon$ between consecutive steps in the conjugate gradient search. Initially it appeared that a tolerance of 0.1 for the RMS gradient was satisfactory, but this was subsequently found to cause problems for clusters containing more than about 60 atoms. The lowest energy structures obtained during the canonical simulation were saved and reoptimized with tolerances of $10^{-4}$ and $10^{-9}$ for the RMS force and the energy difference, respectively. The final energies are accurate to about six decimal places.

Several other techniques were employed in these calculations, namely seeding, freezing and angular moves. Here we used the pair energy per atom, $E(i)$, defined as

$$
E(i)=4 \epsilon \sum_{j \neq i}\left[\left(\frac{\sigma}{r_{i j}}\right)^{12}-\left(\frac{\sigma}{r_{i j}}\right)^{6}\right]
$$

so that the total energy is

$$
E=\frac{1}{2} \sum_{i} E(i)
$$

If the highest pair energy rose above a fraction $\alpha$ of the lowest pair energy then an angular move was employed for the atom in question with all other atoms fixed. $\alpha$ was adjusted to give an acceptance ratio for angular moves of 0.5 and generally converged to between 0.40 and 0.44 . Each angular displacement consisted of choosing random $\theta$ and $\phi$ spherical polar coordinates for the atom in question, taking the origin at the centre of mass and replacing the radius with the maximum value in the cluster.

The two remaining runs for each size consisted of only 200 Monte Carlo steps starting from the global minima obtained for the clusters containing one more and one less atom. When starting from $\mathrm{LJ}_{N-1}$ the $N-1$ atoms were frozen for the first 100 steps, during which only angular moves were attempted for the remaining atom, starting from a random position outside the core. When starting from $\mathrm{LJ}_{N+1}$ the atom with the highest pair energy, $E(i)$, was removed and 200 unrestricted Monte Carlo moves were attempted from the resulting geometry.

The above basin-hopping algorithm shares a common philosophy with our previous approach in which steps were taken directly between minima using eigenvectorfollowing to calculate pathways.26 The latter method is similar to that described recently by Barkema and Mousseau 60 in their search for well-relaxed configurations in glasses. Although the computational expense of transition state searches probably makes this method uncompetitive for global optimization, our study illustrated the possible advantage of working in a space in which only the minima are present. The basin-hopping algorithm differs in that it is applied in configuration space to a transformed surface, rather than in a discrete space of minima, and steps are taken stochastically. The genetic algorithms described by Deaven et al 16 and Niesse and Mayne 30 used conjugate gradient minimization to refine the local minima which comprise the population of structures that are evolved in their procedure. Hence these authors are in effect studying the same transformed surface as described above, but explore it in a rather different manner. We suspect that the success of their methods is at least partly due to the implicit use of the transformed surface $\tilde{E}$.

The transformation of the PES also reduces the barriers to dissociation. Therefore, to prevent evaporation either the cluster can be by placed in a tight-fitting container or the coordinates of the current point in configuration space can be reset to that of the minimum after each successful step. In this paper we used the latter method and then the present approach is essentially the same as the 'Monte Carlo-minimization' algorithm of Li and Scheraga 61 who applied it to search the conformational space of the pentapeptide $\left[\mathrm{Met}^{5}\right]$ enkephalin. A similar method has recently been used by Baysal and Meirovitch 62 to search the conformational space of cyclic polypeptides.
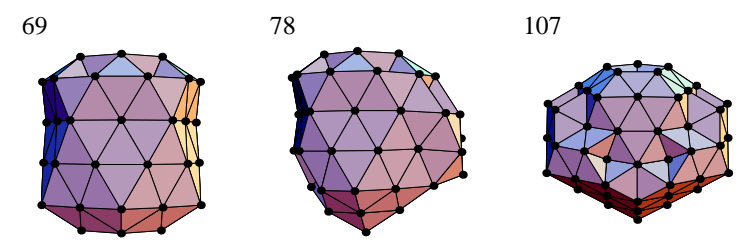

FIG. 3. Lennard-Jones global minima that have not previously been reported.

\section{RESULTS}

The basin-hopping algorithm has successfully located all the lowest known minima up to $\mathrm{LJ}_{110}$, including all the non-icosahedral structures illustrated in Figure 2 (sizes $38,75,76,77,102,103$ and 104) and three new geometries based upon icosahedra illustrated in Figure 3 (sizes 69, 78 and 107). We believe that this is the first time any of the six decahedral global minima have been located by an unbiased algorithm. The total number of searches was fixed in our calculations to provide a simple reference criterion. In fact, most of the global minima were 
found in more than one of the separate Monte Carlo runs. The global minima for the smallest clusters were located within a few steps in each of the seven runs. To give a better idea of how the algorithm performed we will provide some more details for the sizes with non-icosahedral or newly discovered icosahedral global minima.

For $\mathrm{LJ}_{38}$ the truncated octahedron was found in four out of five of the longer unseeded runs; the first success occurred within a thousand Monte Carlo steps on average. Not surprisingly, the global minimum was not located in the shorter runs starting from the structurally unrelated global minima for $N=37$ and 39 .

For $\mathrm{LJ}_{75}$ the global minimum was found in just one of the longer Monte Carlo runs, and again in the short run from the global minimum for $\mathrm{LJ}_{76}$. However, the latter minimum was only found in the short runs seeded from the $\mathrm{LJ}_{75}$ and $\mathrm{LJ}_{77}$ decahedra. Similarly, the $\mathrm{LJ}_{77}$ global minimum was only found in the short run seeded from the $\mathrm{LJ}_{76}$ decahedron. The decahedral global minimum for $\mathrm{LJ}_{75}$ was found in four out of 100 Monte Carlo runs of 5000 steps each, a frequency which fits in quite well with our results for $\mathrm{LJ}_{75}, \mathrm{LJ}_{76}$ and $\mathrm{LJ}_{77}$. A successful run requires an initial geometry which falls within the decahedral catchment area; all the other runs produce the lowest icosahedral minimum after which the decahedron is never found. It would obviously be possible to locate global minima based upon decahedra more efficiently by biasing the starting configuration, but our intention was to analyze the performance of an unbiased algorithm in the present work.

The pattern for $\mathrm{LJ}_{75}-\mathrm{LJ}_{77}$ is repeated for $\mathrm{LJ}_{102}-\mathrm{LJ} J_{103}$. For $\mathrm{LJ}_{102}$ the decahedral global minimum was located in one of the longer Monte Carlo runs and in the short run seeded from the global minimum of $\mathrm{LJ}_{103}$. The decahedral minima for $\mathrm{LJ}_{103}$ and $\mathrm{LJ}_{104}$ were only found in short runs seeded from larger or smaller decahedra. The decahedral global minimum for $\mathrm{LJ}_{102}$ was found in three out of 100 Monte Carlo runs of 5000 steps each.

The three new icosahedral global minima all have an atom missing from a vertex of the underlying Mackay icosahedron (Figure 3). This is a possibility that Northby did not consider in his restricted search of the icosahedral configuration space. The new $\mathrm{LJ}_{69}$ global minimum was found in three of the five longer Monte Carlo runs and in the short run seeded from $\mathrm{LJ}_{70}$. The new global minimum for $\mathrm{LJ}_{78}$ was only found in the short run seeded from $\mathrm{LJ}_{79}$. The new minimum for $\mathrm{LJ}_{107}$ was found in one of the longer Monte Carlo runs and in the short run seeded from $\mathrm{LJ}_{108}$.

We also performed a few preliminary runs for $\mathrm{LJ}_{192}$ and $\mathrm{LJ}_{201}$, sizes at which a complete Marks decahedron and a complete truncated octahedron occur, respectively. For LJ $\mathrm{J}_{192}$ the Marks decahedron has energy -1175.697144 . This structure was not found in $100 \mathrm{MC}$ runs of 10000 steps each; instead the lowest minimum located had an energy of -1174.919801 . For $\mathrm{LJ}_{201}$ the truncated octahedron has energy -1232.731497 . How- ever, we located a structure of energy -1236.124253 which is based upon icosahedral packing. This minimum was found in three out of $50 \mathrm{MC}$ runs of 10000 steps each. For these larger systems greater efficiency could probably be achieved by varying the temperature and other parameters of the MC search.

\section{CONCLUSIONS}

We have presented the resylts of a 'basin-hopping' or 'Monte Carlo-minimization'61 approach to global optimization for atomic clusters bound by the Lennard-Jones potential containing up to 110 atoms. All the lowest known minima were located successfully, including the seven structures based upon fcc or decahedral packing and three new global minima based upon icosahedra. Of the latter ten structures, only the smallest has been located before by an unbiased algorithm, to the best of our knowledge.

The method is based upon a hypersurface deformation in which the potential energy surface (PES) is converted into a set of plateaus each corresponding to a basin of attraction of a local minimum on the original PES. This process removes all the transition state regions but does not affect the energies of the minima. On the original PES, most trajectories that approach the boundary between two basins of attraction are reflected back due to the high potential energy; only if the trajectory is along a transition state valley does passage between basins become likely. In contrast, on the transformed PES it is feasible for the system to hop between basins at any point along the basin boundary which dramatically reduces the time scale for interbasin motion. We speculate that the success of a previous genetic algorithm applied to the same clusters may be at least partly due to the fact that the same space is implicitly considered in that approach. 16

The efficiency of the present approach could doubtless be improved by combining it with various other techniques. The most obvious short-cut would be to start not from initial random configurations but from seeds with either decahedral, icosahedral or fcc morphologies. We have already checked that such biasing is indeed effective, but our aim in the present paper was to gauge the performance of the unbiased algorithm. The temperature at which our Monte Carlo runs were conducted was also not optimized systematically.

Finally, as we noted in the introduction, global optimization for Lennard-Jones clusters at most sizes is a relatively easy task. A more stringent and general test is provided by Morse clusters which exhibit different structural hehaviour as a function of the range of the potential 14, 22 At short range the task is particularly difficult_hecause the PES is very rngged - the number of minima 29 and the barrier heights 63 increase as the range is decreased. 
TABLE I. Global minima of $\mathrm{LJ}_{N}$ for $N \leq 110$. The references in which each minimum was first reported (to the best of our knowledge) are given, and $\dagger$ indicates the present work. We intend to maintain an updated database of energies and coordinates for LJ and Morse clusters on our web site: http://brian.ch.cam.ac.uk

\begin{tabular}{|c|c|c|c|c|c|c|c|}
\hline $\mathrm{N}$ & Point group & Energy $/ \epsilon$ & Ref. & $\mathrm{N}$ & Point group & Energy $/ \epsilon$ & Ret \\
\hline$\overline{2}$ & $D_{\infty h}$ & -1.000000 & 2 & 57 & $C_{s}$ & -288.342625 & 10 \\
\hline 3 & $D_{3 h}$ & -3.000000 & 23 & 58 & $C_{3 v}$ & -294.378148 & 10 \\
\hline 4 & $T_{d}$ & -6.000000 & 2 & 59 & $C_{2 v}$ & -299.738070 & 10 \\
\hline 5 & $D_{3 h}$ & -9.103852 & 2.3 & 60 & $C_{s}$ & -305.875476 & 10 \\
\hline 6 & $O_{h}$ & -12.712062 & 23 & 61 & $C_{2 v}$ & -312.008896 & 10 \\
\hline 7 & $D_{5 h}$ & -16.505384 & 2.3 & 62 & $C_{s}$ & -317.353901 & 10 \\
\hline 8 & $C_{s}$ & -19.821489 & 2 & 63 & $C_{1}$ & -323.489734 & 10 \\
\hline 9 & $C_{2 v}$ & -24.113360 & 2.3 & 64 & $C_{s}$ & -329.620147 & 10 \\
\hline 10 & $C_{3 v}$ & -28.422532 & 2 & 65 & $C_{2}$ & -334.971532 & 12 \\
\hline 11 & $C_{2 v}$ & -32.765970 & 2 & 66 & $C_{1}$ & -341.110599 & 11, \\
\hline 12 & $C_{5 v}$ & -37.967600 & 2 & 67 & $C_{s}$ & -347.252007 & 10 \\
\hline 13 & $I_{h}$ & -44.326801 & 23 & 68 & $C_{1}$ & -353.394542 & 10 \\
\hline 14 & $C_{3 v}$ & -47.845157 & 2 & 69 & $C_{5 v}$ & -359.882566 & 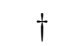 \\
\hline 15 & $C_{2 v}$ & -52.322627 & 2 & 70 & $C_{5 v}$ & -366.892251 & 10 \\
\hline 16 & $C_{s}$ & -56.815742 & 2 & 71 & $C_{5 v}$ & -373.349661 & 10 \\
\hline 17 & $C_{2}$ & -61.317995 & 7 & 72 & $C_{s}$ & -378.637253 & 11 \\
\hline 18 & $C_{5 v}$ & -66.530949 & 2 & 73 & $C_{s}$ & -384.789377 & 10 \\
\hline 19 & $D_{5 h}$ & -72.659782 & 2 & 74 & $C_{s}$ & -390.908500 & 10 \\
\hline 20 & $C_{2 v}$ & -77.177043 & 2 & 75 & $D_{5 h}$ & -397.492331 & 14 \\
\hline 21 & $C_{2 v}$ & -81.684571 & 2 & 76 & $C_{s}$ & -402.894866 & 14 \\
\hline 22 & $C_{s}$ & -86.809782 & 10 & 77 & $C_{2 v}$ & -409.083517 & 14 \\
\hline 23 & $D_{3 h}$ & -92.844472 & 8 & 78 & $C_{s}$ & -414.794401 & - \\
\hline 24 & $C_{s}$ & -97.348815 & 9 & 79 & $C_{2 v}$ & -421.810897 & 10 \\
\hline 25 & $C_{s}$ & -102.372663 & 2 & 80 & $C_{s}$ & -428.083564 & 10 \\
\hline 26 & $T_{d}$ & -108.315616 & 2 & 81 & $C_{2 v}$ & -434.343643 & 10 \\
\hline 27 & $C_{2 v}$ & -112.873584 & 10 & 82 & $C_{1}$ & -440.550425 & 10 \\
\hline 28 & $C_{s}$ & -117.822402 & 10 & 83 & $C_{2 v}$ & -446.924094 & 10 \\
\hline 29 & $D_{3 h}$ & -123.587371 & 2 & 84 & $C_{1}$ & -452.657214 & 10 \\
\hline 30 & $C_{2 v}$ & -128.286571 & 10 & 85 & $C_{3 v}$ & -459.055799 & 10 \\
\hline 31 & $C_{s}$ & -133.586422 & 10 & 86 & $C_{1}$ & -465.384493 & 10 \\
\hline 32 & $C_{2 v}$ & -139.635524 & 10 & 87 & $C_{s}$ & -472.098165 & 10 \\
\hline 33 & $C_{s}$ & -144.842719 & 10 & 88 & $C_{s}$ & -479.032630 & 16 \\
\hline 34 & $C_{2 v}$ & -150.044528 & 10 & 89 & $C_{3 v}$ & -486.053911 & 10 \\
\hline 35 & $C_{1}$ & -155.756643 & 10 & 90 & $C_{s}$ & -492.433908 & 10 \\
\hline 36 & $C_{s}$ & -161.825363 & 10 & 91 & $C_{s}$ & -498.811060 & 10 \\
\hline 37 & $C_{1}$ & -167.033672 & 10 & 92 & $C_{3 v}$ & -505.185309 & 10 \\
\hline 38 & $O_{h}$ & -173.928427 & 13,14 & 93 & $C_{1}$ & -510.877688 & 10 \\
\hline 39 & $C_{5 v}$ & -180.033185 & 10 & 94 & $C_{1}$ & -517.264131 & 10 \\
\hline 40 & $C_{s}$ & -185.249839 & 10 & 95 & $C_{1}$ & -523.640211 & 10 \\
\hline 41 & $C_{s}$ & -190.536277 & 10 & 96 & $C_{1}$ & -529.879146 & 10 \\
\hline 42 & $C_{s}$ & -196.277534 & 10 & 97 & $C_{1}$ & -536.681383 & 10 \\
\hline 43 & $C_{s}$ & -202.364664 & 10 & 98 & $C_{s}$ & -543.642957 & 16 \\
\hline 44 & $C_{1}$ & -207.688728 & 10 & 99 & $C_{2 v}$ & -550.666526 & 10 \\
\hline 45 & $C_{1}$ & -213.784862 & 10 & 100 & $C_{s}$ & -557.039820 & 10 \\
\hline 46 & $C_{2 v}$ & -220.680330 & 10 & 101 & $C_{2 v}$ & -563.411308 & 10 \\
\hline 47 & $C_{1}$ & -226.012256 & 10 & 102 & $C_{2 v}$ & -569.363652 & 15 \\
\hline 48 & $C_{s}$ & -232.199529 & 10 & 103 & $C_{s}$ & -575.766131 & 15 \\
\hline 49 & $C_{3 v}$ & -239.091864 & 10 & 104 & $C_{2 v}$ & -582.086642 & 15 \\
\hline 50 & $C_{s}$ & -244.549926 & 10 & 105 & $C_{1}$ & -588.266501 & 10 \\
\hline 51 & $C_{2 v}$ & -251.253964 & 10 & 106 & $C_{1}$ & -595.061072 & 10 \\
\hline 52 & $C_{3 v}$ & -258.229991 & 10 & 107 & $C_{s}$ & -602.007110 & \\
\hline 53 & $C_{2 v}$ & -265.203016 & 10 & 108 & $C_{s}$ & -609.033011 & 10 \\
\hline 54 & $C_{5 v}$ & -272.208631 & 10 & 109 & $C_{1}$ & -615.411166 & 10 \\
\hline 55 & $I_{h}$ & -279.248470 & 4 & 110 & $C_{s}$ & -621.788224 & 10 \\
\hline 56 & $C_{3 v}$ & -283.643105 & 10 & & & & \\
\hline
\end{tabular}


TABLE II. Lowest energy icosahedral minima at sizes with non-icosahedral global minima.

\begin{tabular}{cccr}
\hline \hline $\mathrm{N}$ & Point group & Energy $/ \epsilon$ & Ref. \\
\hline 38 & $C_{5 v}$ & -173.252378 & 16 \\
75 & $C_{1}$ & -396.282249 & 14 \\
76 & $C_{1}$ & -402.384580 & 12 \\
77 & $C_{1}$ & -408.518265 & 12 \\
102 & $C_{s}$ & -569.277721 & 10 \\
103 & $C_{1}$ & -575.658879 & 10 \\
104 & $C_{s}$ & -582.038429 & 10 \\
\hline \hline
\end{tabular}

\section{ACKNOWLEDGMENTS}

We are grateful to the Royal Society (D.J.W.) and the Engineering and Physical Sciences Research Council (J.P.K.D.) for financial support.

${ }^{1}$ The magnitude of this interest can be gauged by, for example, the fact that there have been over 2600 citations of the classic simulated annealing paper by Kirkpatrick et al., cited below.

${ }^{2}$ M. R. Hoare and P. Pal, Adv. Phys. 20, 161 (1971).

${ }^{3}$ M. R. Hoare and P. Pal, Nature (Physical Science) 230, 645 (1971).

${ }^{4}$ M. R. Hoare and P. Pal, Nature (Physical Science) 236, 35 (1972).

${ }^{5}$ M. R. Hoare and P. Pal, Adv. Phys. 24, 645 (1975).

${ }^{6}$ M. R. Hoare, Adv. Chem. Phys. 40, 49 (1979).

${ }^{7}$ D. L. Freeman and J. D. Doll, J. Chem. Phys. 82, 462 (1985).

8 J. Farges, M. F. de Feraudy, B. Raoult, and G. Torchet, Surf. Sci. 156, 370 (1985)

${ }^{9}$ L. T. Wille, Chem. Phys. Lett. 133, 405 (1987).

10 J. A. Northby, J. Chem. Phys. 87, 6166 (1987).

${ }^{11}$ T. Coleman and D. Shalloway, J. Global Optimization 4, 171 (1994).

${ }^{12}$ G. L. Xue, J.Global Optimization 4, 425 (1994).

13 J. Pillardy and L. Piela, J. Phys. Chem. 99, 11805 (1995).

14 J. P. K. Doye, D. J. Wales, and R. S. Berry, J. Chem. Phys. 103, 4234 (1995).

15 J. P. K. Doye and D. J. Wales, Chem. Phys. Lett. 247, 339 (1995).

${ }^{16}$ D. M. Deaven, N. Tit, J. R. Morris, and K. M. Ho, Chem. Phys. Lett. 256, 195 (1996).

${ }^{17}$ C. J. Tsai and K. D. Jordan, J. Phys. Chem. 97, 11227 (1993).

18 The exact value of this figure should not be be taken too seriously since it depends sensitively on the functional form used to fit Tsai and Jordan's results for the number of minima. The value given was obtained using the form $\exp (a+b N)$, but if one instead uses the form $\exp \left(a+b N+c N^{2}\right)$ the estimate is of the order of $10^{259}$ minima.

19 A. L. Mackay, Acta Cryst. 15, 916 (1962).

${ }^{20}$ R. E. Kunz and R. S. Berry, Phys. Rev. Lett. 71, 3987 (1993).

${ }^{21}$ R. E. Kunz and R. S. Berry, Phys. Rev. E 49, 1895 (1994).

${ }^{22}$ For the larger sizes coexistence in the melting region may occur between the liquid-like state and defective structures based on the global minimum,20,21 and hence molecular dynamics runs at two energies would probably be necessary, first in the melting region and then at a lower energy starting from one of the defective icosahedral structures generated.

${ }^{23}$ L. D. Marks, Phil. Mag. A 49, 81 (1984).

24 These temperatures were calculated using the harmonic superposition method (D. J. Wales, Mol. Phys. , 78, 151 (1993)) in the same manner as described in Ref. 14 for a 75-atom Morse cluster bound by the Morse potential. Note that a $\gamma\left(E^{\prime}\right)_{s}$ incorrectly appears in the relevant equation in Ref. 14.

${ }^{25}$ J. P. K. Doye and D. J. Wales, J. Chem. Phys. 105, 8428 (1996).

${ }^{26}$ J. P. K. Doye and D. J. Wales, Z. Phys. D 40, 194 (1997).

${ }^{27}$ D. R. Nelson and F. Spaepen, Solid State Phys. 42, 1 (1989).

${ }^{28}$ J. P. K. Doye and D. J. Wales, Science 271, 484 (1996).

29 J. P. K. Doye and D. J. Wales, J. Phys. B 29, 4859 (1996).

30 J. A. Niesse and H. R. Mayne, J. Chem. Phys. 105, 4700 (1996).

${ }^{31}$ C. Barrón, S. Gómez, and D. Romero, Appl. Math. Lett. 9, 75 (1996).

32 J. T. Ngo, J. Marks, and M. Karplus, in The Protein Folding Problem and Tertiary Structure Prediction, edited by K. Merz and S. L. Grand (Birkhäuser, Boston, 1994), pp. 433-506.

${ }^{33}$ S. K. Gregurick, M. H. Alexander, and B. Hartke, J. Chem. Phys. 104, 2684 (1996).

34 J. Mestres and G. E. Scuseria, J. Comput. Chem. 16, 729 (1995).

${ }^{35}$ F. H. Stillinger and T. A. Weber, J. Stat. Phys. 52, 1429 (1988).

${ }^{36}$ L. Piela, K. A. Olszewski, and J. Pillardy, J. Mol. Struct. (Theochem) 308, 229 (1994).

37 J. Kostrowicki, L. Piela, B. J. Cherayil, and H. A. Scheraga, J. Phys. Chem. 95, 4113 (1991).

${ }^{38}$ H. A. Scheraga, Int. J. Quant. Chem. 42, 1529 (1992).

${ }^{39}$ F. H. Stillinger and D. K. Stillinger, J. Chem. Phys. 93, 6106 (1990).

${ }^{40}$ T. Head-Gordon, F. H. Stillinger, and D. K. Stillinger, Proc. Natl. Acad. Sci. USA 88, 11076 (1991).

${ }^{41}$ J. Pillardy, K. A. Olszewski, and L. Piela, J. Phys. Chem. 96, 4337 (1992).

42 J. P. K. Doye and D. J. Wales, J. Chem. Soc., Faraday Trans. 93, 4233 (1997).

${ }^{43}$ S. Kirkpatrick, C. D. Gelatt, and M. P. Vecchi, Science 220, 671 (1983).

44 J. Ma, D. Hsu, and J. E. Straub, J. Chem. Phys. 99, 4024 (1993).

45 J. Ma and J. E. Straub, J. Chem. Phys. 101, 533 (1994). 
${ }^{46}$ C. Tsoo and C. L. Brooks, J. Chem. Phys. 101, 6405 (1994).

47 S. Schelstrate and H. Verschelde, J. Phys. Chem. A 42, 1529 (1992).

${ }^{48}$ J. D. Bryngelson, J. N. Onuchic, N. D. Socci, and P. G. Wolynes, Proteins: Structure, Function and Genetics 21, 167 (1995).

${ }^{49}$ A. B. Finnila et al., Chem. Phys. Lett. 219, 343 (1996).

${ }^{50}$ C. D. Maranas and C. A. Floudas, J. Chem. Phys. 97, 7667 (1992).

${ }^{51}$ C. D. Maranas and C. A. Floudas, J. Chem. Phys. 100, 1247 (1994).

${ }^{52}$ A. F. Stanton, R. E. Bleil, and S. Kais, J. Comput. Chem. 18, 594 (1997).

${ }^{53}$ P. Serra, A. F. Stanton, and S. Kais, Phys. Rev. E 55, 1162 (1997).
${ }^{54}$ P. Serra, A. F. Stanton, S. Kais, and R. E. Bleil, J. Chem. Phys. 106, 7170 (1997).

${ }^{55}$ D. Cvijovic and J. Klinowski, Science 267, 664 (1995).

${ }^{56}$ S. D. Hong and M. S. Jhon, Chem. Phys. Lett. 267, 422 (1997)

57 W. H. Press et al., Numerical Recipes (Cambridge University Press, Cambridge, 1986).

58 D. J. Wales, J. Chem. Soc., Faraday Trans. 88, 653 (1992).

${ }^{59}$ D. J. Wales, J. Chem. Soc., Faraday Trans. 89, 1305 (1993).

${ }^{60}$ G. T. Barkema and N. Mousseau, Phys. Rev. Lett. 77, 4358 (1996).

61 Z. Li and H. A. Scheraga, Proc. Natl. Acad. Sci. USA 84, 6611 (1987).

${ }^{62}$ C. Baysal and H. Meirovitch, J. Chem. Phys. 105, 7868 (1996).

${ }^{63}$ D. J. Wales, J. Chem. Phys. 101, 3750 (1994). 